\title{
Bicomplex quantum mechanics: I. The generalized Schrödinger equation
}

\author{
D. Rochon* S. Tremblay ${ }^{\dagger}$ \\ Département de mathématiques et d'informatique \\ Université du Québec à Trois-Rivières \\ C.P. 500 Trois-Rivières, Québec \\ Canada, G9A 5H7
}

\begin{abstract}
We introduce the set of bicomplex numbers $\mathbb{T}$ which is a commutative ring with zero divisors defined by $\mathbb{T}=\left\{w_{0}+w_{1} \mathbf{i}_{1}+w_{2} \mathbf{i}_{\mathbf{2}}+\right.$ $\left.w_{3} \mathbf{j} \mid w_{0}, w_{1}, w_{2}, w_{3} \in \mathbb{R}\right\}$ where $\mathbf{i}_{\mathbf{1}}^{2}=-1, \mathbf{i}_{\mathbf{2}}^{2}=-1, \mathbf{j}^{2}=1, \mathbf{i}_{\mathbf{1}} \mathbf{i}_{\mathbf{2}}=\mathbf{j}=$ $\mathbf{i}_{2} \mathbf{i}_{1}$. We present the conjugates and the moduli associated with the bicomplex numbers. Then we study the bicomplex Schrödinger equation and found the continuity equations. The discrete symmetries of the system of equations describing the bicomplex Schrödinger equation are obtained. Finally, we study the bicomplex Born formulas under the discrete symetries. We obtain the standard Born's formula for the class of bicomplex wave functions having a null hyperbolic angle.
\end{abstract}

PACS: 03.65.-w, 02.10.Hh

\footnotetext{
${ }^{*}$ E-mail: dominic.rochon@uqtr.ca

${ }^{\dagger}$ E-mail: sebastien.tremblay@uqtr.ca
} 


\section{Introduction}

In this paper we investigate the bicomplex Schrödinger equation where bicomplex numbers $\mathbb{T}$ (also called "tetranumbers" in the literature) are defined as the set $\mathbb{T}:=\left\{w_{0} \dot{\mathbf{i}}_{\mathbf{0}}+w_{1} \mathbf{i}_{\mathbf{1}}+w_{2} \mathbf{i}_{\mathbf{2}}+w_{3} \mathbf{j} \mid w_{0}, w_{1}, w_{2}, w_{3} \in \mathbb{R}\right\}$ with

\begin{tabular}{|c||c|c|c|c|}
\hline$\cdot$ & $\mathbf{i}_{0}$ & $\mathbf{i}_{1}$ & $\mathbf{i}_{2}$ & $\mathbf{j}$ \\
\hline \hline $\mathbf{i}_{0}$ & $\mathbf{i}_{0}$ & $\mathbf{i}_{1}$ & $\mathbf{i}_{2}$ & $\mathbf{j}$ \\
\hline $\mathbf{i}_{1}$ & $\mathbf{i}_{1}$ & $-\mathbf{i}_{0}$ & $\mathbf{j}$ & $-\mathbf{i}_{2}$ \\
\hline $\mathbf{i}_{2}$ & $\mathbf{i}_{2}$ & $\mathbf{j}$ & $-\mathbf{i}_{\mathbf{0}}$ & $-\mathbf{i}_{1}$ \\
\hline $\mathbf{j}$ & $\mathbf{j}$ & $-\mathbf{i}_{2}$ & $-\mathbf{i}_{1}$ & $\mathbf{i}_{0}$ \\
\hline
\end{tabular}

We call $\mathbf{i}_{\mathbf{1}}$ and $\mathbf{i}_{\mathbf{2}}$ the imaginary units and we attribute to $\mathbf{j}$ the name of hyperbolic (imaginary) unit. The set of bicomplex numbers is a commutative ring with unit and zero divisors. Hence, contrary to quaternions, bicomplex numbers are commutative with some non-invertible elements situated on the "null cone".

The extension of quantum mechanics beyond the field of complex numbers have been studied by different authors [1, 2, 3, 4, 5]. We know from Frobenius that, in the case of algebra without zero divisor, the investigation must be limited to three fields: real numbers $\mathbb{R}$, complex numbers $\mathbb{C}$ and quaternions $\mathbb{H}$. However, recently, some interest have been deployed to study quantum mechanics beyond the paradigm of algebra without zero divisors [2, 4, 7], principally over hyperbolic numbers $\mathbb{D}$ (also called duplex numbers in the literature). In [4 the author has shown that quantum mechanics over the hyperbolic numbers, called here "hyperbolic quantum mechanics", behaves well 1) in the probabilistic interpretation via the Born's formula, 2 ) for the continuity equation $\partial_{t} P+\nabla \cdot \mathbf{J}=0$ ( where $P$ and $\mathbf{J}$ are respectively the scalar-valued "density" and vector-valued "current") and 3) for the free-particle. However, the main difference between standard quantum mechanics and hyperbolic quantum mechanics comes from the fact that they have different topology on the unit circle. Indeed, the symmetry groups of the unit circle for complex numbers and hyperbolic numbers are respectively $S O(2) \sim S^{1} \subset \mathbb{C}$ and $S O_{\uparrow}(1,1) \sim \mathbb{R} \subset \mathbb{D}$. The consequence of this difference in the topology of the unit circle is that the superposition of states "doesn't hold" in the case of hyperbolic quantum mechanics. For instance, it is well known that in the classical Young's two-slit experiment, the intensity has a sinusoidal pattern. However, in the case of hyperbolic quantum mechanics, intensity is proportional to the hyperbolic cosines [4]. Therefore, the fringe 
pattern cannot be explained by the hyperbolic quantum mechanics. Nevertheless, in [2], it is mentioned that hyperbolic quantum mechanics can be interesting as a new theory of probability waves that can be developed in parallel with standard quantum mechanics [7].

The bicomplex numbers are at the same time a generalization of complex numbers $\mathbb{C}$ and of hyperbolic numbers $\mathbb{D}$. Hence, the "bicomplex quantum mechanics" is some generalization of the standard quantum mechanics and of the hyperbolic quantum mechanics.

In this paper, we investigate the properties of the bicomplex Schrödinger equation. In section 2, we introduce the bicomplex numbers and present the conjugations and the bicomplex moduli of these numbers. Then, in section 3 , we recall some well known results on the standard Schrödinger equation. In section 4 we derive the continuity equations, find the discrete symmetries and introduce the idempotent basis for the bicomplex Schrödinger equation. Finally, in section 5, we introduce the three real moduli for bicomplex numbers and give bicomplex Born formulas. A conclusion is made.

\section{Bicomplex numbers}

The bicomplex numbers are defined as [8, 9, 10]

$$
\mathbb{T}:=\left\{w_{0}+w_{1} \mathbf{i}_{1}+w_{2} \mathbf{i}_{\mathbf{2}}+w_{3} \mathbf{j} \mid w_{0}, w_{1}, w_{2}, w_{3} \in \mathbb{R}\right\},
$$

with the product of imaginary units given in (1.1) i.e., $\mathbf{i}_{\mathbf{0}}:=1$ acts as identity, $\mathbf{i}_{\mathbf{1}}^{2}=\mathbf{i}_{\mathbf{2}}^{2}=-1, \mathbf{j}^{2}=1$ and

$$
\begin{aligned}
\mathbf{i}_{1} \mathbf{i}_{\mathbf{2}} & =\mathbf{i}_{\mathbf{2}} \mathbf{i}_{\mathbf{1}}=\mathbf{j}, \\
\mathbf{i}_{1} \mathbf{j} & =\mathbf{j} \mathbf{i}_{\mathbf{1}}=-\mathbf{i}_{\mathbf{2}}, \\
\mathbf{i}_{2} \mathbf{j} & =\mathbf{j} \mathbf{i}_{\mathbf{2}}=-\mathbf{i}_{\mathbf{1}} .
\end{aligned}
$$

Hence, the bicomplex numbers are commutative. We define the following two subsets $\mathbb{C}\left(\mathbf{i}_{\mathbf{k}}\right) \subset \mathbb{T}$ for $k=1,2$, by $\mathbb{C}\left(\mathbf{i}_{\mathbf{k}}\right):=\left\{x+y \mathbf{i}_{\mathbf{k}} \mid \mathbf{i}_{\mathbf{k}}^{2}=-1\right.$ and $x, y \in$ $\mathbb{R}\}$.

It is also convenient to write the set of bicomplex numbers as

$$
\mathbb{T}=\left\{z_{1}+z_{2} \mathbf{i}_{2} \mid z_{1}, z_{2} \in \mathbb{C}\left(\mathbf{i}_{1}\right)\right\} .
$$

In particular, if we put $z_{1}=x$ and $z_{2}=y \mathbf{i}_{\mathbf{1}}$ with $x, y \in \mathbb{R}$, then we obtain the subalgebra of hyperbolic numbers: $\mathbb{D}=\left\{x+y \mathbf{j} \mid \mathbf{j}^{2}=1, x, y \in \mathbb{R}\right\}$. (Hyperbolic coordinates are naturally introduced in special relativity and serve as space-time coordinates in the Lorentzian's plane, where the noninvertible coordinates correspond to the light cone, and the elements of the form $\mathrm{e}^{\phi \mathbf{j}}$ represent "boosts", [11].) 


\subsection{Conjugates for bicomplex numbers}

Complex conjugation plays an important role both for algebraic and geometric properties of $\mathbb{C}$, as well as in the standard quantum mechanics. For bicomplex numbers, there are three possible conjugations. Let $w \in \mathbb{T}$ and $z_{1}, z_{2} \in \mathbb{C}\left(\mathbf{i}_{1}\right)$ such that $w=z_{1}+z_{2} \mathbf{i}_{\mathbf{2}}$. Then we define the three conjugations as:

$$
\begin{aligned}
& w^{\dagger_{1}}=\left(z_{1}+z_{2} \mathbf{i}_{2}\right)^{\dagger_{1}}:=\bar{z}_{1}+\bar{z}_{2} \mathbf{i}_{2}, \\
& w^{\dagger_{2}}=\left(z_{1}+z_{2} \mathbf{i}_{2}\right)^{\dagger_{2}}:=z_{1}-z_{2} \mathbf{i}_{2}, \\
& w^{\dagger_{3}}=\left(z_{1}+z_{2} \mathbf{i}_{2}\right)^{\dagger_{3}}:=\bar{z}_{1}-\bar{z}_{2} \mathbf{i}_{2},
\end{aligned}
$$

where $\bar{z}_{k}$ is the standard complex conjugate of complex numbers $z_{k} \in \mathbb{C}\left(\mathbf{i}_{1}\right)$. If we say that the bicomplex number $w=z_{1}+z_{2} \mathbf{i}_{\mathbf{2}}=w_{0}+w_{1} \mathbf{i}_{\mathbf{1}}+w_{2} \mathbf{i}_{\mathbf{2}}+w_{3} \mathbf{j}$ has the "signature" $(++++)$, then the conjugations of type 1,2 or 3 of $w$ have, respectively, the signatures $(+-+-),(++--)$ and $(+--+)$.

We can verify easily that each of these conjugates can be expressed in

\begin{tabular}{|c|c|c|c|c|}
\hline 0 & $t_{0}$ & $\dagger_{1}$ & $t_{2}$ & $t_{3}$ \\
\hline$t_{0}$ & $t_{0}$ & $\dagger_{1}$ & $\dagger_{2}$ & $\dagger_{3}$ \\
\hline$\dagger_{1}$ & $\dagger_{1}$ & $t_{0}$ & $\dagger_{3}$ & $t_{2}$ \\
\hline$t_{2}$ & $t_{2}$ & $\dagger_{3}$ & $t_{0}$ & $t_{1}$ \\
\hline$t_{3}$ & $t_{3}$ & $t_{2}$ & $t_{1}$ & $t_{0}$ \\
\hline
\end{tabular}
terms of the two others, i.e. $w^{\dagger_{3}}=\left(w^{\dagger_{1}}\right)^{\dagger_{2}}=\left(w^{\dagger_{2}}\right)^{\dagger_{1}}$, etc. More precisely, under the composition, the conjugates form the following abelian group:

where $w^{\dagger_{0}}:=w \forall w \in \mathbb{T}$.

The three kind of conjugations all have the standard properties of conjugations, i.e.

$$
\begin{aligned}
& (s+t)^{\dagger_{k}}=s^{\dagger_{k}}+t^{\dagger_{k}}, \\
& \left(s^{\dagger_{k}}\right)^{\dagger_{k}}=s, \\
& (s \cdot t)^{\dagger_{k}}=s^{\dagger_{k}} \cdot t^{\dagger_{k}} .
\end{aligned}
$$

for $s, t \in \mathbb{T}$ and $k=0,1,2,3$. The proofs of these properties are rather technical and simple. Nevertheless, let us illustrate the proof for the last 
property in the case of the conjugation of the first kind. Let $s=z_{1}+z_{2} \mathbf{i}_{2}$ and $t=z_{3}+z_{4} \mathbf{i}_{2}$ with $z_{1}, z_{2}, z_{3}, z_{4} \in \mathbb{C}\left(\mathbf{i}_{1}\right)$, then

$$
\begin{aligned}
(s \cdot t)^{\dagger_{1}} & =\left[\left(z_{1} z_{3}-z_{2} z_{4}\right)+\left(z_{1} z_{4}+z_{2} z_{3}\right) \mathbf{i}_{2}\right]^{\dagger_{1}} \\
& =\overline{\left(z_{1} z_{3}-z_{2} z_{4}\right)}+\overline{\left(z_{1} z_{4}+z_{2} z_{3}\right)} \mathbf{i}_{\mathbf{2}} \\
& =\left(\overline{z_{1} z_{3}}-\overline{z_{2} z_{4}}\right)+\left(\overline{z_{1} z_{4}}+\overline{z_{2} z_{3}}\right) \mathbf{i}_{2} \\
& =\left(\bar{z}_{1}+\bar{z}_{2} \mathbf{i}_{\mathbf{2}}\right) \cdot\left(\bar{z}_{3}+\bar{z}_{4} \mathbf{i}_{2}\right) \\
& =s^{\dagger_{1}} \cdot t^{\dagger_{1}} .
\end{aligned}
$$

\section{$2.2 \quad$ The bicomplex moduli}

We know that the product of a standard complex number with his conjugate gives the square of the Euclidean metric in $\mathbb{R}^{2}$. The analog of these, for bicomplex numbers, are the following. Let $z_{1}, z_{2} \in \mathbb{C}\left(\mathbf{i}_{1}\right)$ and $w=z_{1}+z_{2} \mathbf{i}_{\mathbf{2}} \in$ $\mathbb{T}$, then we have that [10]:

$$
\begin{aligned}
|w|_{\mathbf{i}_{1}}^{2} & :=w \cdot w^{\dagger_{2}}=z_{1}^{2}+z_{2}^{2} \in \mathbb{C}\left(\mathbf{i}_{1}\right), \\
|w|_{\mathbf{i}_{2}}^{2} & :=w \cdot w^{\dagger_{1}}=\left(\left|z_{1}\right|^{2}-\left|z_{2}\right|^{2}\right)+2 \operatorname{Re}\left(z_{1} \bar{z}_{2}\right) \mathbf{i}_{\mathbf{2}} \in \mathbb{C}\left(\mathbf{i}_{\mathbf{2}}\right), \\
|w|_{\mathbf{j}}^{2} & :=w \cdot w^{\dagger_{3}}=\left(\left|z_{1}\right|^{2}+\left|z_{2}\right|^{2}\right)-2 \operatorname{Im}\left(z_{1} \bar{z}_{2}\right) \mathbf{j} \in \mathbb{D},
\end{aligned}
$$

where the subscript of the square modulus refers to the subalgebra $\mathbb{C}\left(\mathbf{i}_{1}\right), \mathbb{C}\left(\mathbf{i}_{2}\right)$ or $\mathbb{D}$ of $\mathbb{T}$ in which $w$ is projected. Note that for $z_{1}, z_{2} \in \mathbb{C}\left(\mathbf{i}_{1}\right)$ and $w=$ $z_{1}+z_{2} \mathbf{i}_{\mathbf{2}} \in \mathbb{T}$, we can define the usual norm of $w$ as $|w|=\sqrt{\left|z_{1}\right|^{2}+\left|z_{2}\right|^{2}}=$ $\sqrt{\operatorname{Re}\left(|w|_{\mathbf{j}}^{2}\right)}$.

It is easy to verify that $w \cdot \frac{w^{\dagger_{2}}}{|w|_{\mathbf{i}_{1}}^{2}}=1$. Hence, the inverse of $w$ is given by

$$
w^{-1}=\frac{w^{\dagger_{2}}}{|w|_{\mathbf{i}_{1}}^{2}} .
$$

From this, we find that the set $\mathcal{N C}$ of zero divisors of $\mathbb{T}$, called the null-cone, is given by $\left\{z_{1}+z_{2} \mathbf{i}_{2} \mid z_{1}^{2}+z_{2}^{2}=0\right\}$, which can be rewritten as

$$
\mathcal{N C}=\left\{z\left(\mathbf{i}_{1} \pm \mathbf{i}_{\mathbf{2}}\right) \mid z \in \mathbb{C}\left(\mathbf{i}_{1}\right)\right\} .
$$




\subsection{Exponential function}

Contrary to quaternions, the exponential function is well defined on bicomplex numbers and posses all the standard properties. Hence, for $z_{1}, z_{2} \in$ $\mathbb{C}\left(\mathbf{i}_{1}\right)$ and $w=z_{1}+z_{2} \mathbf{i}_{\mathbf{2}} \in \mathbb{T}$, we have

$$
\mathrm{e}^{w}=\mathrm{e}^{z_{1}+z_{2} \mathbf{i}_{2}}=\mathrm{e}^{z_{1}} \mathrm{e}^{z_{2} \mathbf{i}_{2}}=\mathrm{e}^{z_{1}}\left(\cos z_{2}+\mathbf{i}_{2} \sin z_{2}\right),
$$

corresponding to hyper-polar coordinates. It is easy to see that this is a generalization of the polar coordinates for the complex and the hyperbolic numbers. Indeed, in particular for $z_{1}=\ln r \in \mathbb{R}$ and $z_{2}=\theta \in \mathbb{R}$, we obtain the standard complex polar coordinates. If $z_{1}=\ln \rho \in \mathbb{R}$ and $z_{2}=\phi \mathbf{i}_{\mathbf{1}}$ with $\phi \in \mathbb{R}$, then the equation (2.12) becomes

$$
\begin{aligned}
\mathrm{e}^{z_{1}+z_{2} \mathbf{i}_{2}}=\rho e^{\phi \mathbf{j}} & =\rho\left[\cos \left(\phi \mathbf{i}_{\mathbf{1}}\right)+\mathbf{i}_{\mathbf{2}} \sin \left(\phi \mathbf{i}_{\mathbf{1}}\right)\right] \\
& =\rho\left[\cosh \phi+\mathbf{i}_{\mathbf{2}} \mathbf{i}_{\mathbf{1}} \sinh \phi\right] \\
& =\rho[\cosh \phi+\mathbf{j} \sinh \phi],
\end{aligned}
$$

which corresponds to the hyperbolic polar coordinates used in references [2, 4, 7, 9, 11, 12].

As in the standard case, the bicomplex number $\mathrm{e}^{w}$, is always invertible $\forall w \in \mathbb{T}$. Moreover, we have these useful properties for all the conjugates:

$$
\left(\mathrm{e}^{w}\right)^{\dagger_{k}}=\left(\mathrm{e}^{w^{\dagger} k}\right), k=0,1,2,3 .
$$

\section{The standard Schrödinger's equation}

Before going in the analysis of the bicomplex Schrödinger equation, let us first review some well known results of the standard one-dimensional Schrödinger's equation:

$$
\mathbf{i} \hbar \partial_{t} \psi(x, t)+\frac{\hbar^{2}}{2 m} \partial_{x}^{2} \psi(x, t)-V(x, t) \psi(x, t)=0
$$

where

$$
\psi: \mathbb{R}^{2} \rightarrow \mathbb{C} \text { and } V: \mathbb{R}^{2} \rightarrow \mathbb{R}
$$

First, if we set $\psi=\mathrm{e}^{\alpha(x, t)+\beta(x, t) \mathbf{i}}$ with $\alpha, \beta: \mathbb{R}^{2} \rightarrow \mathbb{R}$, then it is well known that the Schrödinger's equation can be rewritten in a system of two differential equations in terms of the functions $\alpha$ and $\beta$ :

$$
\begin{gathered}
-\hbar \partial_{t} \beta+\frac{\hbar^{2}}{2 m}\left[\partial_{x}^{2} \alpha+\left(\partial_{x} \alpha\right)^{2}-\left(\partial_{x} \beta\right)^{2}\right]-V=0, \\
\partial_{t} \alpha+\frac{\hbar}{2 m}\left[\partial_{x}^{2} \beta+2 \partial_{x} \alpha \partial_{x} \beta\right]=0 .
\end{gathered}
$$


The probability density $P(\psi)$ to find a particle in the state $\psi(x, t)$ is then given by the Born's formula:

$$
P(\psi)=\psi \bar{\psi}=\mathrm{e}^{2 \alpha} .
$$

One other very well known result of the standard Schrödinger's equation is the conservation of the probability current

$$
\partial_{t}(\psi \bar{\psi})+\nabla \cdot \mathbf{J}(\psi)=0
$$

where

$$
\begin{aligned}
J(\psi) & =\frac{\hbar}{2 m \mathbf{i}}\left(\bar{\psi} \partial_{x} \psi-\psi \partial_{x} \bar{\psi}\right) \\
& =\frac{\hbar}{m} \mathrm{e}^{2 \alpha} \partial_{x} \beta .
\end{aligned}
$$

We note that equation (3.2b) and the conservation of the current probability (3.4) coincide. Hence, by decomposing the standard Schrödinger's equation into his real and imaginary parts, one obtain two equations: 3.2a corresponding to an extended version of the Jacobi-Hamilton equation and (3.2b) corresponding to the conservation of the probability current.

\section{The bicomplex Schrödinger equation}

Let us now consider an analog of the one-dimensional standard Schrödinger's equation over the bicomplex space functions:

$$
\mathbf{i}_{1} \hbar \partial_{t} \psi(x, t)+\frac{\hbar^{2}}{2 m} \partial_{x}^{2} \psi(x, t)-V(x, t) \psi(x, t)=0
$$

where

$$
\psi: \mathbb{R}^{2} \rightarrow \mathbb{T} \text { and } V: \mathbb{R}^{2} \rightarrow \mathbb{R}
$$

The choice of the imaginary unit $\mathbf{i}_{\mathbf{1}}$ appearing explicitly in the bicomplex Schrödinger equation is not arbitrary. In the case of hyperbolic quantum mechanics it have been shown that the choice of the hyperbolic imaginary unit $\mathbf{j}$ doesn't yield the superposition principle [4. Hence, in our case, we should choose between the imaginary units $\mathbf{i}_{\mathbf{1}}$ or $\mathbf{i}_{\mathbf{2}}$. In principle there is no major raison to prefer $\mathbf{i}_{\mathbf{1}}$ instead of $\mathbf{i}_{\mathbf{2}}$, however we will see later that imaginary unit $\mathbf{i}_{\mathbf{1}}$ is more appropriated for the decomposition of the bicomplex Schrödinger equation into what we will call the idempotent basis. 
We express the wave function $\psi(x, t)$ into the hyper-polar coordinates as

$$
\psi(x, t)=\mathrm{e}^{z_{1}(x, t)+z_{2}(x, t) \mathbf{i}_{2}},
$$

where

$$
\begin{aligned}
& z_{1}(x, t)=\alpha(x, t)+\beta(x, t) \mathbf{i}_{\mathbf{1}}, \\
& z_{2}(x, t)=\gamma(x, t)+\delta(x, t) \mathbf{i}_{\mathbf{1}},
\end{aligned}
$$

and $\alpha,{ }_{,} \gamma$ and $\delta$ are real functions going from $\mathbb{R}^{2} \rightarrow \mathbb{R}$. Hence, one can decompose the bicomplex Schrödinger equation (4.1) into a system of four differential equations in terms of the four real functions $\alpha, \beta, \gamma$ and $\delta$ :

$$
\begin{gathered}
-\hbar \partial_{t} \beta+\frac{\hbar^{2}}{2 m}\left[\partial_{x}^{2} \alpha+\left(\partial_{x} \alpha\right)^{2}-\left(\partial_{x} \beta\right)^{2}-\left(\partial_{x} \gamma\right)^{2}+\left(\partial_{x} \delta\right)^{2}\right]-V=0, \\
\partial_{t} \alpha+\frac{\hbar}{2 m}\left[\partial_{x}^{2} \beta+2\left(\partial_{x} \alpha \partial_{x} \beta-\partial_{x} \gamma \partial_{x} \delta\right)\right]=0 \\
-\partial_{t} \delta+\frac{\hbar}{2 m}\left[\partial_{x}^{2} \gamma+2\left(\partial_{x} \alpha \partial_{x} \gamma-\partial_{x} \beta \partial_{x} \delta\right)\right]=0 \\
\partial_{t} \gamma+\frac{\hbar}{2 m}\left[\partial_{x}^{2} \delta+2\left(\partial_{x} \alpha \partial_{x} \delta+\partial_{x} \beta \partial_{x} \gamma\right)\right]=0
\end{gathered}
$$

We remark that when $\gamma \rightarrow 0$ and $\delta \rightarrow 0$ the system of equations (4.4) goes to the system (3.2) of the standard Schrödinger's equation.

\subsection{The bicomplex continuity equations}

In this section, we derive the continuity equations for the bicomplex Schr ödinger equation. For that, we rewrite the bicomplex Schrödinger equation under the four kind of conjugations:

$$
\begin{aligned}
\mathbf{i}_{1} \hbar \partial_{t} \psi+\frac{\hbar^{2}}{2 m} \partial_{x}^{2} \psi-V \psi & =0, \\
-\mathbf{i}_{1} \hbar \partial_{t} \psi^{\dagger_{1}}+\frac{\hbar^{2}}{2 m} \partial_{x}^{2} \psi^{\dagger_{1}}-V \psi^{\dagger_{1}} & =0, \\
\mathbf{i}_{1} \hbar \partial_{t} \psi^{\dagger_{2}}+\frac{\hbar^{2}}{2 m} \partial_{x}^{2} \psi^{\dagger_{2}}-V \psi^{\dagger_{2}} & =0, \\
-\mathbf{i}_{1} \hbar \partial_{t} \psi^{\dagger_{3}}+\frac{\hbar^{2}}{2 m} \partial_{x}^{3} \psi^{\dagger_{3}}-V \psi^{\dagger_{3}} & =0 .
\end{aligned}
$$


Let us first consider equations (4.5a) and (4.5b). Multiplying (4.5a) by $\psi^{\dagger_{1}}$ and (4.5b) by $\psi$, and subtracting these two equations, one obtain

$$
\mathbf{i}_{1} \partial_{t}\left(\psi \psi^{\dagger_{1}}\right)+\frac{\hbar}{2 m}\left(\psi^{\dagger_{1}} \partial_{x}^{2} \psi-\psi \partial_{x}^{2} \psi^{\dagger_{1}}\right)=0,
$$

which can be rewritten into the continuity equation

$$
\partial_{t}\left(\psi \psi^{\dagger 1}\right)+\nabla \cdot \mathbf{J}_{1}(\psi)=0
$$

where

$$
\begin{aligned}
J_{1}(\psi) & =\frac{\hbar}{2 m \mathbf{i}_{\mathbf{1}}}\left(\psi^{\dagger_{1}} \partial_{x} \psi-\psi \partial_{x} \psi^{\dagger_{1}}\right), \\
& =\frac{\hbar}{m} \mathrm{e}^{2\left(\alpha+\gamma \mathbf{i}_{\mathbf{2}}\right)} \partial_{x}\left(\beta+\delta \mathbf{i}_{\mathbf{2}}\right) .
\end{aligned}
$$

These calculations can be done for all pair of equations in the set of equations (4.5). However, one can construct a continuity equation only if the sign of the imaginary unit $\mathbf{i}_{\mathbf{1}}$, in front of each equation, are opposite into the pair of equations that we consider. Indeed, it is not possible to obtain a continuity equation from the pair of equations (4.5a) and (4.5c) or (4.5b) and (4.5d). Therefore, we find three other continuity equations (for a total of four):

$$
\begin{gathered}
\partial_{t}\left(\psi \psi^{\dagger_{3}}\right)+\nabla \cdot \mathbf{J}_{2}(\psi)=0, \\
J_{2}(\psi)=\frac{\hbar}{2 m \mathbf{i}_{\mathbf{1}}}\left(\psi^{\dagger_{3}} \partial_{x} \psi-\psi \partial_{x} \psi^{\dagger_{3}}\right), \\
=\frac{\hbar}{m} \mathrm{e}^{2(\alpha+\delta \mathbf{j})} \partial_{x}(\beta-\gamma \mathbf{j}) . \\
\partial_{t}\left(\psi^{\dagger_{2}} \psi^{\dagger_{1}}\right)+\nabla \cdot \mathbf{J}_{3}(\psi)=0, \\
J_{3}(\psi)=\frac{\hbar}{2 m \mathbf{i}_{\mathbf{1}}}\left(\psi^{\dagger_{1}} \partial_{x} \psi^{\dagger_{2}}-\psi^{\dagger_{2}} \partial_{x} \psi^{\dagger_{1}}\right), \\
=\frac{\hbar}{m} \mathrm{e}^{2(\alpha-\delta \mathbf{j})} \partial_{x}(\beta+\gamma \mathbf{j}) .
\end{gathered}
$$




$$
\begin{aligned}
\partial_{t}\left(\psi^{\dagger_{2}} \psi^{\dagger_{3}}\right)+ & \nabla \cdot \mathbf{J}_{4}(\psi)=0, \\
J_{4}(\psi) & =\frac{\hbar}{2 m \mathbf{i}_{\mathbf{1}}}\left(\psi^{\dagger_{3}} \partial_{x} \psi^{\dagger_{2}}-\psi^{\dagger_{2}} \partial_{x} \psi^{\dagger_{3}}\right), \\
& =\frac{\hbar}{m} \mathrm{e}^{2\left(\alpha-\gamma \mathbf{i}_{2}\right)} \partial_{x}\left(\beta-\delta \mathbf{i}_{\mathbf{2}}\right) .
\end{aligned}
$$

However, on can verify that these four continuity equations are an over determined system of equations. Indeed, if we conjugate equation (4.7) by $\dagger_{2}$, we obtain equation (4.13) where $\mathbf{J}_{4}=\left(\mathbf{J}_{1}\right)^{\dagger_{2}}$. In the same way, if we conjugate equation (4.9) by $\dagger_{1}$ we obtain equation (4.11) with $\mathbf{J}_{3}=\left(\mathbf{J}_{2}\right)^{\dagger_{1}}$. Therefore, we have two independent continuity equations. Let us choose $\mathbf{J}_{1}$ and $\mathbf{J}_{2}$ as the "basis" for the currents.

The continuity equations (4.7) is in fact equivalent to the equations (4.4b) and (4.4d). In the same way, the continuity equations (4.9) is equivalent to the equations (4.4b) and (4.4c). Therefore, equation (4.4a) corresponds to an extended version of the Hamilton-Jacobi equation of the standard case and the system (4.4b), (4.4c) and (4.4d) are equivalent to the continuity equations (4.7) and (4.9) expresed in terms of $\mathbf{J}_{1}$ and $\mathbf{J}_{2}$ only.

\subsection{Discrete symmetries of the bicomplex Schrödinger equation}

The system of equations (4.4) posses a 8-dimensional discrete group, leaving the solution set of the system invariant. These discrete symmetry group is given by

$$
\begin{aligned}
& \hat{P}_{0}=I d \quad \hat{P}_{1}=\left\{\begin{array}{lll}
\gamma & \rightarrow & -\gamma \\
\delta & \rightarrow & -\delta
\end{array}\right. \\
& \hat{P}_{2}=\left\{\begin{array}{rrr}
\gamma & \rightarrow & -\delta \mathbf{i}_{2} \\
\delta & \rightarrow & \gamma \mathbf{i}_{2}
\end{array} \quad \hat{P}_{3}=\left\{\begin{array}{rrr}
\gamma & \rightarrow & \delta \mathbf{i}_{\mathbf{2}} \\
\delta & \rightarrow & -\gamma \mathbf{\mathbf { i } _ { 2 }}
\end{array}\right.\right. \\
& \hat{P}_{4}=\left\{\begin{array}{rrr}
\gamma & \rightarrow & \delta \mathbf{i}_{\mathbf{1}} \\
\delta & \rightarrow & -\gamma \mathbf{i}_{\mathbf{1}}
\end{array} \quad \hat{P}_{5}=\left\{\begin{array}{rrr}
\gamma & \rightarrow & -\delta \mathbf{i}_{\mathbf{1}} \\
\delta & \rightarrow & \gamma \mathbf{i}_{\mathbf{1}}
\end{array}\right.\right. \\
& \hat{P}_{6}=\left\{\begin{array}{l}
\gamma \rightarrow \gamma \mathbf{j} \\
\delta \rightarrow \delta \mathbf{j}
\end{array} \quad \hat{P}_{7}=\left\{\begin{array}{l}
\gamma \rightarrow-\gamma \mathbf{j} \\
\delta \rightarrow-\delta \mathbf{j}
\end{array} .\right.\right.
\end{aligned}
$$

Note that functions $\alpha(x, t)$ and $\beta(x, t)$, of the bicomplex wave function $\psi(x, t)$, are not transformed under these discrete symmetry group. 
Let us mention some remarks about these transformations. First, the group of symmetry (4.15) is an abelian group with $\hat{P}_{n}^{2}=I d$ for $n=$ $0,1, \ldots, 7$. Second, the set given by $\left\{\hat{P}_{0}, \hat{P}_{1}, \hat{P}_{2}, \hat{P}_{3}\right\}$ is a subgroup of the symmetry group and is isomorphic to the group of conjugates (2.5) for the bicomplex numbers. Finally, we remark that the discrete operators $\hat{P}_{4}, \hat{P}_{5}, \hat{P}_{6}$ and $\hat{P}_{7}$ act on an arbitrary bicomplex number $w$ exactly as the discrete operators $\hat{P}_{0}, \hat{P}_{1}, \hat{P}_{2}$ and $\hat{P}_{3}$, respectively, i.e.

$$
\hat{P}_{n+4}(w)=\hat{P}_{n}(w), \text { for } n=0,1,2,3 \forall w \in \mathbb{T} .
$$

Hence we have in fact a "fundamental subgroup", for the symmetry group, given by $\left\{\hat{P}_{0}, \hat{P}_{1}, \hat{P}_{2}, \hat{P}_{3}\right\}$.

Let us now apply these symmetries on the system of equations equivalent to (4.4), i.e. the system of equations consisting of (4.4a) and the two continuity equations (4.7) and (4.9). We already know that equation (4.4a) is invariant under the symmetries. Let us now look how the continuity equations (4.7) and (4.9) are transformed under these symmetries. For that, we only have to calculate the action of the symmetry operators on $\psi$ (since $\mathbf{J}_{1}$ and $\mathbf{J}_{2}$ are expressible in term of $\psi$ ). We find that

$$
\hat{P}_{1}(\psi)=\psi^{\dagger_{2}}, \hat{P}_{2}(\psi)=\psi_{+}, \hat{P}_{3}(\psi)=\psi_{-},
$$

where the functions $\psi_{+}$and $\psi_{-}$are functions in the $\mathbb{C}\left(\mathbf{i}_{\mathbf{1}}\right)$-space given by

$$
\psi_{ \pm}=\mathrm{e}^{z_{1} \mp z_{2} \mathbf{i}_{1}}=\mathrm{e}^{(\alpha \pm \delta)+(\beta \mp \gamma) \mathbf{i}_{1}} .
$$

From these calculations, we find that $\hat{P}_{1}$ transforms equations (4.7) and (4.9) into equations (4.13) and (4.11), respectively. Under the discrete symmetry $\hat{P}_{2}$, the equations (4.7) and (4.9) are both transformed into the continuity equation

$$
\partial_{t}\left(\psi_{+} \bar{\psi}_{+}\right)+\nabla \cdot \mathbf{J}\left(\psi_{+}\right)=0
$$

where

$$
\begin{aligned}
J\left(\psi_{+}\right) & =\frac{\hbar}{2 m \mathbf{i}_{1}}\left(\bar{\psi}_{+} \partial_{x} \psi_{+}-\psi_{+} \partial_{x} \bar{\psi}_{+}\right) \\
& =\frac{\hbar}{m} \mathrm{e}^{2(\alpha+\delta)} \partial_{x}(\beta-\gamma) .
\end{aligned}
$$

Finally, under $\hat{P}_{3}$, the equations (4.7) and (4.9) are both transformed into the continuity equation

$$
\partial_{t}\left(\psi_{-} \bar{\psi}_{-}\right)+\nabla \cdot \mathbf{J}\left(\psi_{-}\right)=0,
$$




$$
\begin{aligned}
J\left(\psi_{-}\right) & =\frac{\hbar}{2 m \mathbf{i}_{1}}\left(\bar{\psi}_{-} \partial_{x} \psi_{-}-\psi_{-} \partial_{x} \bar{\psi}_{-}\right) \\
& =\frac{\hbar}{m} \mathrm{e}^{2(\alpha-\delta)} \partial_{x}(\beta+\gamma) .
\end{aligned}
$$

Hence, under the symmetry operators, we have recover the two continuity equations (4.13) and (4.11), dropped previously since respectively equivalent to (4.7) and (4.9). Moreover, we have found two new continuity equations (4.19) and (4.21), associated with two real currents $\mathbf{J}\left(\psi_{+}\right)$and $\mathbf{J}\left(\psi_{-}\right)$.

Note that it is possible to express the bicomplex wave function $\psi$ in terms of $\psi_{+}$and $\psi_{-}$by using what is called the idempotents basis. Indeed, for all bicomplex numbers, one can pass from the real basis $\left\{1, \mathbf{i}_{\mathbf{1}}, \mathbf{i}_{\mathbf{2}}, \mathbf{j}\right\}$ to the complex (in $\mathbf{i}_{\mathbf{1}}$ ) basis $\left\{\mathbf{e}_{\mathbf{1}}, \mathbf{e}_{\mathbf{2}}\right\}$, where $\mathbf{e}_{\mathbf{1}}=\frac{1+\mathbf{j}}{2}, \mathbf{e}_{\mathbf{2}}=\frac{1-\mathbf{j}}{2}$ (in fact $\mathbf{e}_{\mathbf{2}}$ can be rewritten in term of $\mathbf{e}_{\mathbf{1}}$, i.e. $\mathbf{e}_{\mathbf{2}}=\left(\mathbf{e}_{\mathbf{1}}\right)^{\dagger_{1}}=\left(\mathbf{e}_{\mathbf{1}}\right)^{\dagger_{2}}$, but $\left.\mathbf{e}_{\mathbf{2}} \neq\left(\mathbf{e}_{\mathbf{1}}\right)^{\dagger_{3}}\right)$. The elements $\mathbf{e}_{\mathbf{1}}, \mathbf{e}_{\mathbf{2}}$ having the following properties:

$$
\left(\mathbf{e}_{1}\right)^{2}=\mathbf{e}_{1},\left(\mathbf{e}_{2}\right)^{2}=\mathbf{e}_{2}, \mathbf{e}_{1} \mathbf{e}_{2}=0 .
$$

Every bicomplex numbers $z_{1}+z_{2} \mathbf{i}_{\mathbf{2}}, z_{1}, z_{2} \in \mathbb{C}\left(\mathbf{i}_{\mathbf{1}}\right)$, can be expressed in the idempotent basis as

$$
z_{1}+z_{2} \mathbf{i}_{\mathbf{2}}=\left(z_{1}-z_{2} \mathbf{i}_{\mathbf{1}}\right) \mathbf{e}_{\mathbf{1}}+\left(z_{1}+z_{2} \mathbf{i}_{\mathbf{1}}\right) \mathbf{e}_{\mathbf{2}} .
$$

Moreover, the bicomplex exponential can be rewritten as follows

$$
\mathrm{e}^{z_{1}+z_{2} \mathbf{i}_{\mathbf{2}}}=\mathrm{e}^{z_{1}-z_{2} \mathbf{i}_{1}} \mathbf{e}_{\mathbf{1}}+\mathrm{e}^{z_{1}+z_{2} \mathbf{i}_{1}} \mathbf{e}_{\mathbf{2}} .
$$

In the same way, we can express the wave function in the idempotent basis as

$$
\psi=\psi_{+} \mathbf{e}_{\mathbf{1}}+\psi_{-} \mathbf{e}_{\mathbf{2}} .
$$

Hence, using the idempotent basis, we can rewrite the bicomplex Schrödinger equation (4.1) in the form

$$
\left(\mathbf{i}_{\mathbf{1}} \hbar \partial_{t} \psi_{+}+\frac{\hbar^{2}}{2 m} \partial_{x}^{2} \psi_{+}-V \psi_{+}\right) \mathbf{e}_{\mathbf{1}}+\left(\mathbf{i}_{\mathbf{1}} \hbar \partial_{t} \psi_{-}+\frac{\hbar^{2}}{2 m} \partial_{x}^{2} \psi_{-}-V \psi_{-}\right) \mathbf{e}_{\mathbf{2}}=0,
$$

which can be decomposed into the following two standard Schrödinger's equations (complex in $\left.\mathbf{i}_{\mathbf{1}}\right)$ :

$$
\mathbf{i}_{1} \hbar \partial_{t} \psi_{ \pm}+\frac{\hbar^{2}}{2 m} \partial_{x}^{2} \psi_{ \pm}-V \psi_{ \pm}=0
$$

Associated with these equations, it is now obvious to see that we have the continuity equations (4.19) and (4.21) written, respectively, in terms of the real currents $J\left(\psi_{ \pm}\right)$given by (4.20) and (4.22). 


\section{The bicomplex Born formula}

In the case of the standard Schrödinger's equation (linear and homogeneous) it is well known that the continuous symmetries of (3.1), acting on a solution $\psi$ only, are

$$
\begin{aligned}
& \psi \rightarrow \lambda \psi, \lambda \in \mathbb{C}, \\
& \psi \rightarrow \psi+\phi(\phi \text { an other solution of the Schrödinger's eq. }),
\end{aligned}
$$

corresponding, respectively, to a dilation of the wave function and the superposition principle. In quantum mechanics dilation is used for the normalization of the wave function and the superposition principle is one fundamental property.

In particular, the dilation can be expressed as a rotation of the wave function when $\lambda=\mathrm{e}^{i \theta}$. These particular symmetry plays an important role in quantum mechanics since it is invariant under the Born's formula.

For the bicomplex Schrödinger equation we still have the dilation (with $\lambda \in \mathbb{T}$ ) and the superposition principle (where $\psi$ and $\phi$ are bicomplex functions) as continuous symmetries, since equation (4.1) is linear and homogeneous. However, in addition, we have the discrete symmetries given in (4.15). In this section, we want to study the bicomplex discrete symmetries for bicomplex Born formulas.

\subsection{Definitions of the real moduli}

In order to obtain some bicomplex Born formula from our bicomplex wave function $\psi(x, t)$, let us define the following three real moduli (see [10]):

1) For $s, t \in \mathbb{T}$, we define the first modulus as $|\cdot|_{\mathbf{1}}:=\|\left.\cdot\right|_{\mathbf{i}_{\mathbf{1}}} \mid$. This modulus has the following properties:

a) $|\cdot|_{1}: \mathbb{T} \rightarrow \mathbb{R}$

b) $|s|_{\mathbf{1}} \geq 0$ with $|s|_{\mathbf{1}}=0$ iff $s \in \mathcal{N C}$,

c) $|s \cdot t|_{\mathbf{1}}=|s|_{\mathbf{1}} \cdot|t|_{\mathbf{1}}$.

From this definition, we can rewrite this real pseudo-modulus in a much practical point of view as

$$
|w|_{\mathbf{1}}=\left|z_{1}^{2}+z_{2}^{2}\right|^{1 / 2}
$$

for $w=z_{1}+z_{2} \mathbf{i}_{\mathbf{2}}$ with $z_{1}, z_{2} \in \mathbb{C}\left(\mathbf{i}_{1}\right)$. Moreover, it is also useful to express $|\cdot|_{\mathbf{1}}$, in terms of our three bicomplex conjugates, i.e.

$$
|w|_{\mathbf{1}}=\sqrt[4]{w w^{\dagger_{1}} w^{\dagger_{2}} w^{\dagger_{3}}} .
$$


2) For $s, t \in \mathbb{T}$, we define the second modulus as $|\cdot|_{\mathbf{2}}:=|| \cdot\left|\mathbf{i}_{\mathbf{2}}\right|$. This modulus has the same properties as $|\cdot|_{\mathbf{1}}$. Indeed we can rewrite $|w|_{\mathbf{2}}$ as

$$
|w|_{2}=\left|z_{1}^{2}+z_{2}^{2}\right|^{1 / 2}
$$

where $w=z_{1}+z_{2} \mathbf{i}_{1}$ with $z_{1}, z_{2} \in \mathbb{C}\left(\mathbf{i}_{2}\right)$. Hence, the first and the second pseudo-modulus are the same.

3) For $s, t \in \mathbb{T}$, we define the third modulus as $|\cdot|_{\mathbf{3}}:=|| \cdot|\mathbf{j}|$. This modulus has the following properties:

a) $|\cdot|_{3}: \mathbb{T} \rightarrow \mathbb{R}$

b) $|s|_{\mathbf{3}} \geq 0$ with $|s|_{\mathbf{3}}=0$ iff $s=0$,

c) $|s+t|_{\mathbf{3}} \leq|s|_{\mathbf{3}}+|t|_{\mathbf{3}}$,

d) $|s \cdot t|_{\mathbf{3}} \leq \sqrt{2}|s|_{\mathbf{3}} \cdot|t|_{\mathbf{3}}$.

We note that

(i) $|w|_{\mathbf{j}}=\left|z_{1}-z_{2} \mathbf{i}_{\mathbf{1}}\right| \mathbf{e}_{\mathbf{1}}+\left|z_{1}+z_{2} \mathbf{i}_{\mathbf{1}}\right| \mathbf{e}_{\mathbf{2}} \in \mathbb{D} \forall w=z_{1}+z_{2} \mathbf{i}_{\mathbf{2}} \in \mathbb{T}$,

(ii) $|s \cdot t|_{\mathbf{j}}=|s|_{\mathbf{j}}|t|_{\mathbf{j}} \forall s, t \in \mathbb{T}$.

From this definition, we can rewrite the modulus $|\cdot|_{\mathbf{3}}$ as

$$
|w|_{\mathbf{3}}=\sqrt{\left|z_{1}\right|^{2}+\left|z_{2}\right|^{2}}
$$

for $w=z_{1}+z_{2} \mathbf{i}_{\mathbf{2}}$ with $z_{1}, z_{2} \in \mathbb{C}\left(\mathbf{i}_{\mathbf{1}}\right)$. Hence, we see that in fact $|\cdot|_{\mathbf{3}}$ is simply the Euclidean metric of $\mathbb{R}^{4}$, i.e.

$$
|w|_{\mathbf{3}}=|w|=\sqrt{\operatorname{Re}\left(|w|_{\mathbf{j}}^{2}\right)}
$$

\subsection{Invariance under the discrete symmetries for the real moduli}

Let us first calculate the real moduli for the bicomplex wave function $\psi(x, t)$. We obtain the following "bicomplex Born formulas":

$$
\begin{aligned}
& |\psi|_{\mathbf{1}}^{2}=|\psi|_{\mathbf{2}}^{2}=\mathrm{e}^{2 \alpha}, \\
& |\psi|_{\mathbf{3}}^{2}=\mathrm{e}^{2 \alpha} \cosh (2 \delta)=\mathrm{e}^{2 \alpha}\left(1+\frac{(2 \delta)^{2}}{2 !}+\frac{(2 \delta)^{4}}{4 !}+\cdots\right) .
\end{aligned}
$$


For $|\psi|_{\mathbf{1}}^{2}$, we find the same result as in the standard case (3.3) and $|\psi|_{\mathbf{3}}^{2}$ is some kind of hyperbolic perturbation of the standard case when $\delta(x, t)$ is small.

Let us now consider the invariance of $|\psi|_{\mathbf{k}}^{2}(k=1,2,3)$ under the discrete symmetries. To illustrate that, we first consider the operator $\hat{P}_{1}$. A new wave function $\tilde{\psi}$ is obtained by applying the symmetry operator $\hat{P}_{1}$ on $\psi$. Then by calculating the result on $|\tilde{\psi}|_{1}^{2}$, we find

$$
\begin{aligned}
|\tilde{\psi}|_{\mathbf{1}}^{2}:=\left|\hat{P}_{1} \psi\right|_{\mathbf{1}}^{2} & =\sqrt{\left(\hat{P}_{1} \psi\right)\left(\hat{P}_{1} \psi\right)^{\dagger_{1}}\left(\hat{P}_{1} \psi\right)^{\dagger_{2}}\left(\hat{P}_{1} \psi\right)^{\dagger_{3}}} \\
& =\sqrt{\psi^{\dagger_{2}} \psi^{\dagger_{3}} \psi \psi^{\dagger_{1}}} \\
& =|\psi|_{\mathbf{1}}^{2}=\mathrm{e}^{2 \alpha} .
\end{aligned}
$$

Therefore, $|\psi|_{1}^{2}$ is invariant under $\hat{P}_{1}$. Performing these calculations for all the real moduli, under all the discrete symmetries, we obtain

$$
\left|\hat{P}_{1} \psi\right|_{\mathbf{k}}^{2}=|\psi|_{\mathbf{k}}^{2}= \begin{cases}\mathrm{e}^{2 \alpha} & \text { if } k=1,2 \\ \mathrm{e}^{2 \alpha} \cosh (2 \delta) & \text { if } k=3\end{cases}
$$

and

$$
\begin{aligned}
\left|\hat{P}_{2} \psi\right|_{\mathbf{k}}^{2} & =\mathrm{e}^{2 \delta}|\psi|_{\mathbf{k}}^{2}=\mathrm{e}^{2(\alpha+\delta)} \\
\left|\hat{P}_{3} \psi\right|_{\mathbf{k}}^{2} & =\mathrm{e}^{-2 \delta}|\psi|_{\mathbf{k}}^{2}=\mathrm{e}^{2(\alpha-\delta)}
\end{aligned}
$$

for $k=1,2,3$.

It is now easy to prove that the bicomplex Born formulas will be preserved under all our discrete symmetries (4.15) if and only if the wave function $\psi$ has the form

$$
\psi(x, t)=\mathrm{e}^{\alpha(x, t)+\beta(x, t) \mathbf{i}_{1}+\gamma(x, t) \mathbf{i}_{2}},
$$

i.e. if and only if $\delta(x, t)=0$. Moreover, we have the following result for $|\cdot|_{3}^{2}$ :

Theorem 1 Let $\psi$ be a bicomplex wave function given by $\psi(x, t)=$ $\mathrm{e}^{\alpha(x, t)+\beta(x, t) \mathbf{i}_{1}+\gamma(x, t) \mathbf{i}_{2}+\delta(x, t) \mathbf{j}}=\psi_{+}(x, t) \mathbf{e}_{\mathbf{1}}+\psi_{-}(x, t) \mathbf{e}_{\mathbf{2}}$. Then,

$$
|\psi(x, t)|_{\mathbf{3}}^{2}=\frac{\left|\psi_{+}\right|^{2}+\left|\psi_{-}\right|^{2}}{2} .
$$

In particular, if the standard wave functions $\psi_{+}(x, t)$ and $\psi_{-}(x, t)$ are normalized we have that

$$
|\psi(x, t)|_{\mathbf{3}}^{2}=\frac{P_{1}+P_{2}}{2} \in[0,1]
$$


where $P_{1}$ and $P_{2}$ are respectively the density probability of $\psi_{+}(x, t)$ and $\psi_{-}(x, t)$.

Proof. The proof of this theorem is obtained using the following analog of the Pythagoras Theorem for bicomplex numbers (see [8]):

$$
\left|z_{1}+z_{2} \mathbf{i}_{\mathbf{2}}\right|^{2}=\left|\frac{z_{1}-z_{2} \mathbf{i}_{\mathbf{1}}}{\sqrt{2}}\right|^{2}+\left|\frac{z_{1}+z_{2} \mathbf{i}_{\mathbf{1}}}{\sqrt{2}}\right|^{2} \forall z_{1}+z_{2} \mathbf{i}_{\mathbf{2}} \in \mathbb{T},
$$

to the bicomplex wave function $\psi(x, t)$.

We are now ready to summarize our results with this following corollary:

Corollary 1 Let $\psi$ be a bicomplex wave function given by $\psi(x, t)=$ $\mathrm{e}^{\alpha(x, t)+\beta(x, t) \mathbf{i}_{1}+\gamma(x, t) \mathbf{i}_{\mathbf{2}}}=\psi_{+}(x, t) \mathbf{e}_{\mathbf{1}}+\psi_{-}(x, t) \mathbf{e}_{\mathbf{2}}$. Then,

$$
|\psi|^{2}=|\psi|_{\mathbf{1}}^{2}=|\psi|_{\mathbf{2}}^{2}=|\psi|_{\mathbf{3}}^{2}=\sqrt{\psi \psi^{\dagger_{1}} \psi^{\dagger_{2}} \psi^{\dagger_{3}}}=\frac{\left|\psi_{+}\right|^{2}+\left|\psi_{-}\right|^{2}}{2}=\mathrm{e}^{2 \alpha}
$$

where $|\psi|^{2}$ gives the standard Born's formula and is invariant under all the discrete symmetries (4.15) of the bicomplex Schrödinger equation.

The fact that hyperbolic angle of the exponential is zero in $\psi$, i.e. we have to consider $\delta(x, t)=0$ in Corollary 1 , do not means that hyperbolic part of the wave function do not play any role. Indeed, the wave function can be explicitly rewritten as

$$
\begin{aligned}
\psi(x, t) & =\mathrm{e}^{\alpha(x, t)+\beta(x, t) \mathbf{i}_{\mathbf{1}}+\gamma(x, t) \mathbf{i}_{2}} \\
& =\mathrm{e}^{\alpha}\left(\cos \beta \cos \gamma+\mathbf{i}_{\mathbf{1}} \sin \beta \cos \gamma+\mathbf{i}_{\mathbf{2}} \cos \beta \sin \gamma+\mathbf{j} \sin \beta \sin \gamma\right)
\end{aligned}
$$

Hence, the wave function considered in Corollary 1 is really a bicomplex function.

\section{Conclusion}

In this paper we have introduced the bicomplex numbers and some bicomplex conjugates and moduli associated with these numbers. Then we have study the bicomplex Schrödinger equation where we have found the bicomplex continuity equations. Moreover we have shown that, under some discrete symmetries of the system of four equations of the bicomplex Schrödinger equation, the bicomplex continuity equations can be transformed into real 
continuity equations associated with the currents $J\left(\psi_{ \pm}\right)$. These two real currents are in fact associated with the bicomplex Schrödinger equation written in terms of the idempotent basis. Finally, we have shown that it is possible to obtain some specific generalization of the Born's formula for a class of wave functions with a null hyperbolic angle. This class of wave functions are completely invariant under all the discrete symmetries founded.

\section{Acknowledgments}

The research of D.R. was supported by CRSNG of Canada. The research of S.T. was supported by a Postdoctoral Fellowship from FQRNT du Québec. The main part of the work here was written while S.T. was visiting the Faculty of Nuclear Sciences and Physical Engineering of the Czech Technical University in Prague. He thanks Professor Pelantová for her hospitality. 


\section{References}

[1] Adler S.L., Quaternionic Quantum Mechanics and Quantum Fields, 1995, Oxford University Press, New York.

[2] Khrennikov A., 2003, Adv. App. Cliff. Alg. 13 No. 1, 1.

[3] Horwitz L.P., 1996, Found. Phys. 26, No. 6, 851.

[4] Kocik J., 1999, Internat. J. Theoret. Phys. 38, No. 8, 2221.

[5] Finkelstein F.G., 1962, et al. J. Math Phys. 3.

[6] Frobenius F.G., 1878, Jo. Reine Angew. Mat. 84, 1.

[7] Khrennikov A., 2003, Ann. Phys.(8) 12, No. 10, 575.

[8] Price G.B., 1991, An Introduction to Multicomplex Spaces and Functions, Marcel Dekker Inc., New York.

[9] Rochon D., 2001, Doctoral thesis, Université de Montréal.

[10] Rochon D. and Shapiro M., On algebraic properties of bicomplex and hyperbolic numbers, Anal. Univ. Oradea (to appear).

[11] Sobczyk G., 1995, Col. Math. Jour. 26, No 4.

[12] Rochon D., A Bicomplex Riemann Zeta Function, Tokyo J. Math. (to appear).

[13] Cohen-Tannoudji C., Diu B., and Laloë F., 1977, Mécanique quantique, Hermann, Paris.

[14] LeBellac M., 2003, Physique quantique, CNRS, Paris.

[15] Rochon D., 2000, Fractals 8, No. 4, 355. 